\title{
CAD DESIGN AND CONTROL OF TRIPLE INVERTED-PENDULUMS SYSTEM
}

\author{
Mustafa Turki Hussein
}

Email: eng.mustafa.turky@uobabylon.edu.iq

Mechanical Engineering Department / University of Babylon

\begin{abstract}
This work is aimed to study the dynamic behavior and control of the triple invertedpendulum system. A nonlinear dynamic model of the inverted-pendulums fixed on a cart, based on CAD model is developed. The Lagrange equation is used to obtain the nonlinear dynamic models of the system. The dynamic model is then linearized around operating point. An augmented dynamic model using the linearized model is also derived. Two control approaches are used to stabilize the pendulums in vertical position. First approach: State Feedback Control based on the linearized model is used to generate the input force control to stabilize the system. Second approach: Model Predictive Control is designed based on augmented dynamic Model to control the motion of the system. In order to verify the developed model and the chosen controller gains several simulations for different carts' paths are carried out. Several 3D animations are also presented to verify the usefulness of the designed CAD model and the controllers. As a future work: the 3D model of the triple inverted-pendulum system gives a valuable resource for virtual reality work. Beside, another advanced control approach can be applied on the derived dynamic model.
\end{abstract}

Keywords: Inverted-pendulum, CAD models, State Feedback control, Model Predictive control, 3D-animation.

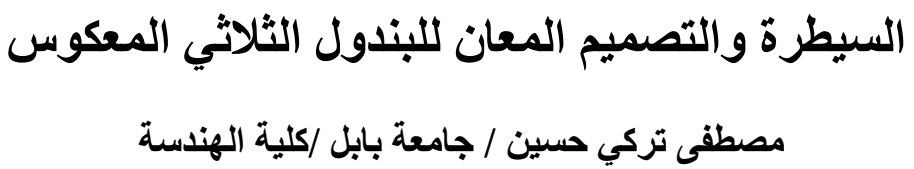

الخلاصة

الهدف من هذا العمل هو دراسة السلوك الديناميكي والسيطرة على نظام متكون من بندول ثلاثي معكوس مثبت على

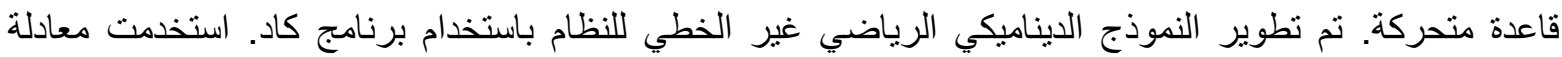

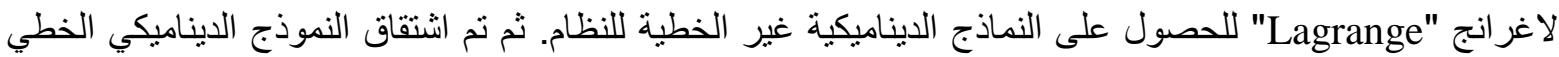

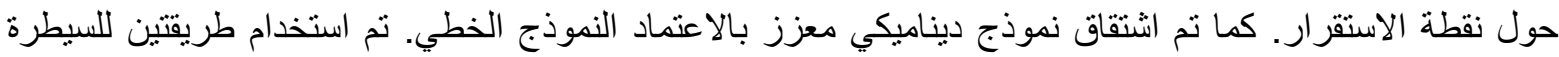

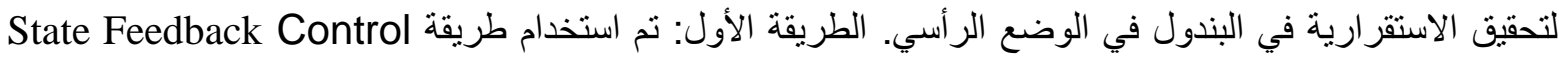

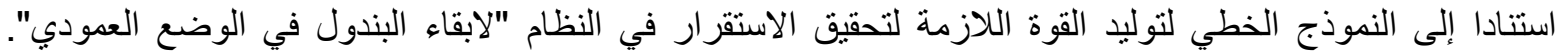

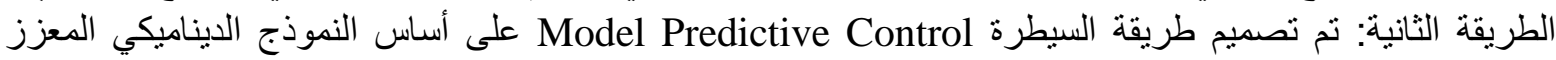

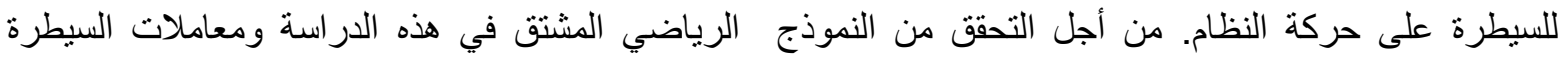

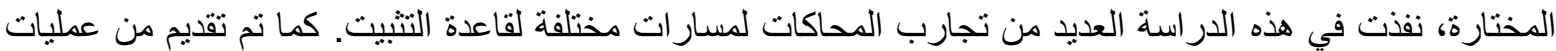

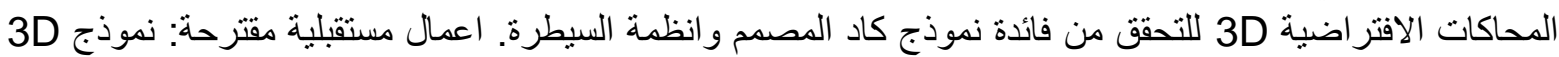
لنظام البندول الثناثي المقلوب يعطي مصدرا قيما للعمل بالواقع الافتراضي. إلى جانب ذللك، يمكن تطبيق نهج سيطرة الفئ

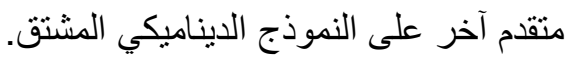
الكلمات المفتاحية: البندول المعكوس، نموذج الإنج معالج بالكومبيوتر، نظام سيطرة، نظام سيطرة تخميني،المحاكات الافتر اضية ثلاثية الابعاد المادية 


\section{NOMENCLATURES}

$L \quad$ Generalized coordinates Lagrangian

Q Generalized force vector

$q \quad$ The generalized coordinates vector

$r \quad$ Cart position on the horizontal axis $X_{0}$

$\vartheta_{i} \quad$ Inclination of each pendulum from the vertical axis $Y_{0}, i=1,2,3$

$\gamma_{i} \quad$ The difference in the angles for the second and the third pendulums, $i=1,2$

$T \quad$ Total kinetic energy of the system

$P \quad$ Total potential energy of the system

$T_{i} \quad$ Kinetic energy of each element of the system, $i=0,1,2,3$

$P_{i} \quad$ Potential energy of each element of the system, $i=0,1,2,3$

$m_{\tilde{i}} \quad$ Mass of each element of the system, $i=0,1,2,3$

$L_{i} \quad$ Length of each pendulum, $i=1,2,3$

$l_{i} \quad$ Center of gravity of each pendulum, $i=1,2,3$

$g$ Gravitational acceleration

$u \quad$ Input force

$I_{i} \quad$ Mass moment of inertia of each pendulum, $i=1,2,3$

$M \quad$ Total Mass Matrix

F Total Coriolis and Centrifugal matrix

$G \quad$ Total Gravitational effect matrix

$H \quad$ Total generalized force matrix

$M_{i} \quad$ Model parameters, $i=1,2, \cdots, 36$

$X \quad$ The generalized state vector

$T_{r} \quad$ Transformation matrix

$A_{0} \quad$ The generalized state matrix

$B$ o The generalized input matrix

A The transformed state matrix

$B \quad$ The transformed input matrix

C The transformed output matrix

D The transformed feedforward matrix

$x \quad$ The transformed state vector

$y$ The output vector

$K_{s} \quad$ State feedback controller gains vector

$\hat{x} \quad$ Estimated transformed state vector

$I$ The performance index for the state feedback controller

$Q_{s} \quad$ The weighting state matrix

$R \quad$ The weighting input matrix

$P_{s} \quad$ The solution of the Riccati equation for the state feedback controller

$S \quad$ The solution of the Riccati equation for the state observer

$A_{d} \quad$ The transformed discrete state matrix

$B_{d} \quad$ The transformed discrete input matrix

$C_{d} \quad$ The transformed discrete output matrix

$k \quad$ The discrete next step symbol

$N_{c} \quad$ Control Horizon of model predictive controller

$x_{\text {aug }}$ Augmented state vector

$A_{\text {aug }}$ Augmented state matrix

$B_{\text {aug }}$ Augmented input matrix 
$C_{\text {aug }} \quad$ Augmented output matrix

$K_{m p c}$ Model predictive controller gains vector

$\rho \quad$ Material density

$r_{d} \quad$ Desired cart position

\section{INTRODUCTION}

The inverted-pendulum system has the characteristics of non-linear, unstable and non-minimum phase dynamics hence the balancing of an inverted-pendulum is an ideal problem in the field of control theory which considerably interests in many fields such as mechanics, physics, and applied mathematics [Brisilla and Sank 2015]. Due to nonlinearity and instability challenging control objectives; Inverted-pendulum systems have used as excellent test-rigs for control theories. This system has two equilibrium points, one of which is stable while the other is unstable. The stable equilibrium point represents the point where the system swings about with decreasing amplitude until it comes to rest if it is released from any position other than precisely vertical. Therefore, the stable equilibrium point needs no control action to be achieved, thus it is uninteresting from a control point of view. The unstable state of the system in which the pendulum points strictly vertical and requires a control input force in order to keep this position.

The inverted-pendulum system is used effectively to illustrate several ideas in linear control theory as stabilization of unstable systems [Wang 2011]. Furthermore, the inverted-pendulum systems and due to their nonlinear nature are used to illustrate many ideas in the nonlinear control field such as: variable control structure, back-stepping control, nonlinear state observers, task oriented control, swinging up control, and nonlinear model reduction [Roose et al., 2017]. The planar inverted-pendulum carried on a cart is the main control idea of the inverted-pendulum system, and has several types such as single inverted-pendulum, double inverted-pendulum and triple invertedpendulum. The control problem of the pendulum system can be divided in general into two problems such as stabilizing and swing-up.

The controllability of the non-zero friction joint (damped) of triple inverted-pendulum is investigated in [Su and Woodham 2003]. The work is aimed to canceling the uncontrollable poles and zeros which can unstabilize the transfer function of the system. A symbolic manipulation for the dynamic model is done in order to calculate the transfer function and a numerical result is also presented. [Gmiterko and Grossman 2009] presented an automatized procedure to derive n-link inverted-pendulum dynamic model. The procedure is incorporating the Maple software for deriving the dynamic model symbolically. A numerical example is also addressed and the LQR control approach is applied for double-inverted pendulum resulted from the numerical example. The motion control of a triple inverted-pendulum on a cart is presented in [Glück et. al. 2013], where two degrees-of-freedom controller scheme is used in order to accomplish the swing-up problem. The scheme contains a nonlinear feedforward controller and an optimal feedback controller. The proposed controller is validated using an experimental test-rig. [Zhang et. al. 2015] proposed a cloud genetic algorithm + PID neural network to control triple inverted-pendulum. Furthermore, a 3D animation model based on Solidworks MATLAB and LabVIEW is also illustrated. The CGA-PIDNN controller validated using the simulation results and 3D animation model. An alternative representation of fractional Lagrangian for modeling of triple inverted-pendulum is presented in [Escamilla et. al. 2016]. The dynamic model of the system is derived based on the fractional derivative definitions in order to simplify the modeling process. A numerical simulation using the representations is done.

In this paper, the design and control of triple inverted-pendulum system is presented. The main objectives of the present work are as follow:

- 3D CAD design using Solidworks of triple inverted-pendulum system, 
- Mathematical modeling of the system,

- $\quad$ Design of state feedback and Model Predictive controllers.

The CAD software "Solidworks" is used to design the system in order to simplify the modeling process. The dynamic model of triple inverted-pendulum under study is derived using the Lagrange equations by implementing the parameters produced from the CAD model. The model linearized around upward operating point for the sake of State Feedback controller design. The linearized model is then discretized and augmented in order to design a Model Predictive Controller (MPC). The Laguerre function is used in the MPC to generate the required input force to control the motion of the system. The graphical programming LabVIEW Software is used to design the controllers' gains for each of the controller type, and also to simulate the controlled motion.

The rest of the paper is organized as follow: in section 2, Triple Inverted-Pendulum model description is presented, after that the nonlinear dynamic model and the linearized model is addressed in section 2.1. Later controller design is explained 'section 3', the State Feedback method and the Model Predictive Control method are detailed in 3.1 and 3.2 respectively. The simulation results are illustrated in section 4. Conclusions and future work suggestions are written in section 5 .

\section{MODELING OF TRIPLE INVERTED-PENDULUM ON A CART}

The triple inverted-pendulum system is a nonlinear under actuated system, furthermore triple inverted-pendulum on a cart represent a challenging control design problem. In this section the dynamic modeling of triple inverted-pendulum on a cart is derived. The system under study is graphically shown in Fig. 1. The dynamic equations of motion are derived using one of the possible ways which is the Lagrange equations:

$\frac{d}{d t} \frac{\partial L}{\partial \dot{q}}-\frac{\partial L}{\partial q}=Q$

Here $L=T-P$ is a Lagrangian. $Q$ is a vector of generalized forces acting in the direction of motion coordinates $q$ and not accounted for; in formulation of kinetic energy $T$ and potential energy $P$. The generalized coordinates vector $q$ is defined as $q=\left[r \vartheta_{1} \vartheta_{2} \vartheta_{3}\right]^{T}$.

Kinetic and potential energies of the system are given by the sum of energies of its individual components (a cart and three pendulums) [Tsachouridis 1999]:

$T=T_{0}+T_{1}+T_{2}+T_{3}$

$P=P_{0}+P_{1}+P_{2}+P_{3}$

Kinetic and Potential energies of the cart is given by;

$T_{0}=\frac{1}{2} m_{0} \dot{r}^{2}, \quad P_{0}=0$.

Kinetic and Potential energies of the first, second and third pendulums can be calculated as

$T_{1}=\frac{1}{2} m_{1}\left[\left(\dot{r}+l_{1} \dot{\vartheta}_{1} \cos \vartheta_{1}\right)^{2}+\left(l_{1} \dot{\vartheta}_{1} \sin \vartheta_{1}\right)^{2}\right]+\frac{1}{2} I_{1} \dot{\vartheta}_{1}^{2}$,

$P_{1}=m_{1} g l_{1} \cos \vartheta_{1}$, 


$$
\begin{aligned}
& T_{2}=\frac{1}{2} m_{2}\left[\left(\dot{r}+L_{1} \dot{\vartheta}_{1} \cos \vartheta_{1}+l_{2} \dot{\vartheta}_{2} \cos \vartheta_{2}\right)^{2}+\left(L_{1} \dot{\vartheta}_{1} \sin \vartheta_{1}+l_{2} \dot{\vartheta}_{2} \sin \vartheta_{2}\right)^{2}\right]+\frac{1}{2} I_{2} \dot{\vartheta}_{2}^{2}, \\
& P_{2}=m_{2} g\left(L_{1} \cos \vartheta_{1}+l_{2} \cos \vartheta_{2}\right), \\
& T_{3}=\frac{1}{2} m_{3}\left[\left(\dot{r}+L_{1} \dot{\vartheta}_{1} \cos \vartheta_{1}+L_{2} \dot{\vartheta}_{2} \cos \vartheta_{2}+l_{3} \dot{\vartheta}_{3} \cos \vartheta_{3}\right)^{2}+\left(L_{1} \dot{\vartheta}_{1} \sin \vartheta_{1}+L_{2} \dot{\vartheta}_{2} \sin \vartheta_{2}+\right.\right. \\
& \left.\left.l_{3} \dot{\vartheta}_{3} \sin \vartheta_{3}\right)^{2}\right]+\frac{1}{2} I_{2} \dot{\vartheta}_{2}^{2} \\
& P_{3}=m_{3} g\left(L_{1} \cos \vartheta_{1}+L_{2} \cos \vartheta_{2}+l_{3} \cos \vartheta_{3}\right) .
\end{aligned}
$$

\subsection{Nonlinear Dynamic Model}

According the above derived Eqs. (2-5) thus the Lagrangian of the three inverted-pendulum system is given by

$$
\begin{aligned}
L=T-P= & \frac{1}{2}\left(m_{0}+m_{1}+m_{2}+m_{3}\right) \dot{r}^{2}+\frac{1}{2}\left(m_{1} l_{1}^{2}+m_{2} L_{1}^{2}+m_{3} L_{1}^{2}+I_{1}\right) \dot{\vartheta}_{1}^{2}+\frac{1}{2}\left(m_{2} l_{2}^{2}\right. \\
& \left.+m_{3} L_{2}^{2}+I_{2}\right) \dot{\vartheta}_{2}^{2}+\frac{1}{2}\left(m_{3} l_{3}^{2}+I_{3}\right) \dot{\vartheta}_{3}^{2}+\left(m_{1} l_{1}+m_{2} L_{1}+m_{3} L_{1}\right) \cos \left(\vartheta_{1}\right) \dot{r} \dot{\vartheta}_{1} \\
& +\left(m_{2} l_{2}+m_{3} L_{2}\right) \cos \left(\vartheta_{2}\right) \dot{r} \dot{\vartheta}_{2}+\left(m_{2} L_{1} l_{2}+m_{3} L_{1} L_{2}\right) \cos \left(\vartheta_{1}-\vartheta_{2}\right) \dot{\vartheta}_{1} \dot{\vartheta}_{2} \\
& +m_{3} l_{3} \cos \left(\vartheta_{3}\right) \dot{r} \dot{\vartheta}_{3}+m_{3} L_{1} l_{3} \cos \left(\vartheta_{1}-\vartheta_{3}\right) \dot{\vartheta}_{1} \dot{\vartheta}_{3}+m_{3} L_{2} l_{3} \cos \left(\vartheta_{2}-\vartheta_{3}\right) \dot{\vartheta}_{2} \dot{\vartheta}_{3} \\
& -\left(m_{1} l_{1}+m_{2} L_{1}+m_{3} L_{1}\right) g \cos \left(\vartheta_{1}\right)-\left(m_{2} l_{2}+m_{3} L_{2}\right) g \cos \left(\vartheta_{2}\right) \\
& -l_{3} m_{3} g \cos \left(\vartheta_{3}\right) .
\end{aligned}
$$

Applying the Lagrangian (6) into the Lagrangian function (1), the dynamic equations of motion for the triple inverted-pendulum on a cart system can be written in a compact matrix form and given by

$$
M(q) \ddot{q}+F(q \cdot \dot{q}) \dot{q}+G(q) q=H u
$$

$M(q)=\left[\begin{array}{cccc}M_{1} & M_{2} \cos \vartheta_{1} & M_{3} \cos \vartheta_{2} & M_{4} \cos \vartheta_{3} \\ M_{9} \cos \vartheta_{1} & M_{10} & M_{11} \cos \left(\vartheta_{1}-\vartheta_{2}\right) & M_{12} \cos \left(\vartheta_{1}-\vartheta_{3}\right) \\ M_{18} \cos \vartheta_{2} & M_{19} \cos \left(\vartheta_{1}-\vartheta_{2}\right) & M_{20} & M_{21} \cos \left(\vartheta_{2}-\vartheta_{3}\right) \\ M_{28} \cos \vartheta_{3} & M_{29} \cos \left(\vartheta_{1}-\vartheta_{3}\right) & M_{30} \cos \left(\vartheta_{2}-\vartheta_{3}\right) & M_{31}\end{array}\right]$

$F(q, \dot{q})$

$=\left[\begin{array}{cccc}M_{5} & M_{6} \sin \left(\vartheta_{1}\right) \dot{\vartheta}_{1} & M_{7} \sin \left(\vartheta_{2}\right) \dot{\vartheta}_{2} & M_{8} \sin \left(\vartheta_{3}\right) \dot{\vartheta}_{3} \\ 0 & M_{13} & M_{14} \sin \left(\vartheta_{1}-\vartheta_{2}\right) \dot{\vartheta}_{2}+M_{15} & M_{16} \sin \left(\vartheta_{1}-\vartheta_{3}\right) \dot{\vartheta}_{3} \\ 0 & M_{22} \sin \left(\vartheta_{1}-\vartheta_{2}\right) \dot{\vartheta}_{1}+M_{23} & M_{24} & M_{25} \sin \left(\vartheta_{2}-\vartheta_{3}\right) \dot{\vartheta}_{3}+M_{26} \\ 0 & M_{33} \sin \left(\vartheta_{1}-\vartheta_{3}\right) \dot{\vartheta}_{1} & M_{35} \sin \left(\vartheta_{2}-\vartheta_{3}\right) \dot{\vartheta}_{2}+M_{36} & M_{32}\end{array}\right]$

$G(q)=\left[\begin{array}{cccc}0 & 0 & 0 & 0 \\ 0 & M_{17} & 0 & 0 \\ 0 & 0 & M_{27} & 0 \\ 0 & 0 & 0 & M_{34}\end{array}\right]$, and $H=\left[\begin{array}{llll}1 & 0 & 0 & 0\end{array}\right]^{T}$.

\section{Linearized State-Space Dynamic Equations of Motion}

The dynamic equations derived in (2.1) section "Eq. (Error! Reference source not found.)" describe the system's motion and they are clearly nonlinear. For the sake of the controller design 
the dynamic equations are linearized around the equilibrium point

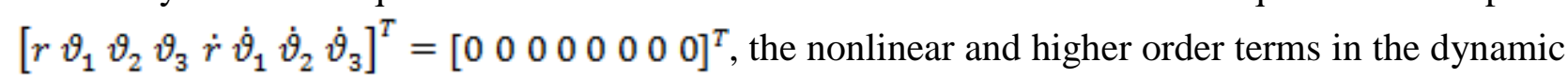
model are neglected. This process leads to the following state-space system

$\dot{X}(t)=A_{o} X(t)+B_{o} u(t)$

Where

$A_{o}=\left[\begin{array}{cc}0_{4 \times 4} & I_{4 \times 4} \\ -M(0)^{-1} G & -M(0)^{-1} F(0,0)\end{array}\right]_{8 \times 8}$, and $B_{o}=\left[\begin{array}{c}0_{4 \times 1} \\ M(0)^{-1} H\end{array}\right]_{8 \times 1}$.

are the state and input matrices respectively, and the state vector is $X(t)=\left[r \vartheta_{1} \vartheta_{2} \vartheta_{3} \dot{r} \dot{\vartheta}_{1} \dot{\vartheta}_{2} \dot{\vartheta}_{3}\right]^{T}$. The outputs of the system in the model are the difference in the angles of the links as shown in Fig. 1, a similarity transformation [Gu et. al. 2013] is defined as follows: $x(t)=T_{r} X(t)=\left[r \vartheta_{1} \gamma_{1} \gamma_{2} \dot{r} \dot{\vartheta}_{1} \dot{\gamma}_{1} \dot{\gamma}_{2}\right]^{T}$.

Here $\gamma_{1}=\vartheta_{2}-\vartheta_{1}, \gamma_{2}=\vartheta_{3}-\vartheta_{2}$, and $T_{r}=\left[\begin{array}{cccccccc}1 & 0 & 0 & 0 & 0 & 0 & 0 & 0 \\ 0 & 1 & 0 & 0 & 0 & 0 & 0 & 0 \\ 0 & -1 & 1 & 0 & 0 & 0 & 0 & 0 \\ 0 & 0 & -1 & 1 & 0 & 0 & 0 & 0 \\ 0 & 0 & 0 & 0 & 1 & 0 & 0 & 0 \\ 0 & 0 & 0 & 0 & 0 & 1 & 0 & 0 \\ 0 & 0 & 0 & 0 & 0 & -1 & 1 & 0 \\ 0 & 0 & 0 & 0 & 0 & 0 & -1 & 1\end{array}\right]$.

Using the transformed states yield the state matrices (10) of the system as: $A=T_{r} A_{\odot} T_{r}^{-1}$ and $B=T_{r} B_{o}$. The output of the system will be in this case the position of the cart and the angles of each link with respect to the link before, $y(t)=C x(t)=\left[r \vartheta_{1} \gamma_{1} \gamma_{2}\right]^{T}$ as shown in Fig. 1.

\section{CONTROLLER DESIGN}

In this section the regulation problem of triple inverted-pendulum is addressed in this article. The main goal of the control system defined in this section is stabilizing the states of closed-loop system around an unstable equilibrium point. Therefore, controller task is to maintain the position of the cart at the specific $r$ position while the pendulum is balanced in upright position. The controller of the system for the regulation problem is designed based on the linear model although the dynamics model of triple inverted pendulum in Eq. (Error! Reference source not found.) are non-linear. In this section and for the sake of validation of the designed model; two types of controllers for the triple-inverted pendulum under study are proposed.

\section{State Feedback Controller}

The dynamic equations of the triple inverted pendulum under study in state form (10) are used to design the feedback gain matrix by linear quadratic regulator (LQR). This method has an advantage over the pole placement method because it allows the feedback gain matrix to be determined that will result in the minimum amount of energy being required to stabilize the system [Franklin et. al. 2014]. The block diagram of the state feedback controller is shown in Fig. 2. The input force according to state feedback control law is given by: 
$u=-K_{s} \hat{x}(t)$

which minimizes the performance index:

$$
I=\int_{0}^{\infty}\left(x^{T} Q_{s} x+u^{T} R u\right) d t .
$$

Where $K_{s}$ is the state feedback controller gains vector, $\widehat{x}$ is the estimated states of the system, $Q$ is a positive semi-definite real matrix and $R$ is a positive definite real matrix. By adjusting the value of the matrix $R$, the amount of control input can be varied. Increasing $R$ will result in less energy being used to control the system [Dorf and Bishop 2016]. The matrix $Q_{s}$ determines the amount of weighting or importance assigned to each of the state variables. A larger value puts more emphasis on that particular variable. The full-order estimator is constructed to estimate the state vector of the system. The selection of the estimator gain matrix $L_{s}$ is also based on LQR. The observer dynamic equation is expressed as:

$\hat{\hat{x}}(t)=A \hat{x}+B u+L_{s}(y-C \hat{x})$.

There are many different design techniques used to design the observer gain matrices [Franklin et. al. 2014]. Here the gains of the controller and the full state observer are found by minimizing a linear quadratic performance index $I$, which leads to solving the algebraic Riccati equation:

$$
A^{T} P_{s}+P_{s} A+Q_{s}-P_{s} B R^{-1} B^{T} P_{s}=0 .
$$

And for the observer the following equation is used:

$$
A S+S A^{T}+Q_{s}-S C^{T} R^{-1} C S=0 .
$$

\section{Model Predictive Control (MPC)}

The main design objective of MPC is to compute a trajectory of a future input or control variable $u$ to optimize the future response of the plant output $y$. The optimization is implemented within a limited time window by giving plant states information at the start of the time window. The basic idea of solving this problem using MPC is to find a way to construct the optimal control trajectory. The control sequence will be computed for a specific time interval called Control Horizon $N_{c}$; it will determine the progress of the system for a longer time interval called the Prediction Horizon [Wang 2009].

First the state-space system dynamic will be discretized using zero order hold with sampling period. The resulted system can be expressed as:

$$
\begin{aligned}
x(k+1) & =A_{d} x(k)+B_{d} u(k) . \\
y(k) & =c_{d} x(k) .
\end{aligned}
$$

In Eq. (16), $x(k)$ is the discrete system states, $A_{d}$ is $8 \times 8$ discrete system matrix, $B_{d}$ is $8 \times 1$ discrete input matrix, and $C_{d}$ is $4 \times 8$ discrete output matrix.

Taking a difference operation on both sides of (16), obtain that: $x(k+1)-x(k)=A_{d}(x(k)-x(k-1))+B_{d}(u(k)-u(k-1)) ; \quad$ by denoting the difference of state variable as $\Delta x(k+1)=x(k+1)-x(k), \Delta x(k)=x(k)-x(k-1)$, and the control variable by: $\Delta u(k)=u(k)-u(k-1)$. With this transformation, the difference of the state-space equation is: 
$\Delta x(k+1)=A_{d} x(k)+B_{d} \Delta u(k)$.

The system now can be augmented in a way to connect $\Delta x(k)$ to the output $y(k)$. To do so, a new state-space model is chosen to be:

$$
\begin{aligned}
& \overbrace{\left[\begin{array}{r}
\Delta x(k+1) \\
y(k+1)
\end{array}\right]}^{x_{\text {aug }}(k+1)}=\overbrace{\left[\begin{array}{cc}
A_{d} & 0 \\
C_{d} A_{d} & I
\end{array}\right]}^{A_{\text {aug }}} \overbrace{\left[\begin{array}{r}
\Delta x(k) \\
y(k)
\end{array}\right]}^{x_{\text {aug }}(k)}+\overbrace{\left[\begin{array}{c}
B_{d} \\
C_{d} B_{d}
\end{array}\right]}^{B_{\text {aug }}} \Delta u(k), \\
& y(k)=\overbrace{\left[\begin{array}{ll}
0 & I
\end{array}\right]}^{c_{\text {aug }}}\left[\begin{array}{c}
\Delta x(k) \\
y(k)
\end{array}\right] .
\end{aligned}
$$

In Eq. (18), $x_{\text {aug }}(k)$ augmented system states, $A_{\text {aug }}$ is $12 \times 12$ augmented system matrix, $B_{\text {aug }}$ is $12 \times 1$ input matrix, and $C_{\text {aug }}$ is $4 \times 12$ output matrix.

The aim now is to use MPC Controller in order to compute the control input force sequence $\Delta u(k)$, [Takashi and Shimada 2017], which is then applied to the system through state feedback to keep the system states in (Error! Reference source not found.) bounded and stable. According to Laguerre function which is used in this work the control input can be written as:

$\Delta u(k)=-K_{\text {mpc }} x_{\text {aug }}(k)$.

Here

$K_{m p c}=\Lambda(0)^{T} \Omega^{-1} \Psi$.

with

$$
\begin{gathered}
\Lambda(0)^{T}=\sqrt{1-a^{2}}\left(1-a a^{2}-a^{3} \cdots(-1)^{N_{c}-1} a^{N_{c}-1}\right), \\
\Omega=\sum_{m=1}^{N_{p}} \phi(m) Q \phi(m)^{T}+R_{L^{*}} \\
\Psi=\sum_{m=1}^{N_{p}} \phi(m) Q A_{\text {aug }}^{m}, \phi(m)^{T}=\sum_{i=0}^{m-1} A_{\text {aug }}^{m-i-1} B_{\text {aug }} \Gamma(i)^{T}, \text { and } \Gamma(i)^{T}=\left(\gamma_{1}(i) \cdots \gamma_{N_{e}}(i)\right) .
\end{gathered}
$$

Using the control gain feedback the controlled system becomes

$$
\begin{array}{r}
x_{\text {aug }}(k+1)=\left(A_{\text {aug }}-B_{\text {aug }} K_{\text {mpo }}\right)+B_{\text {aug }} \Delta u(k) . \\
y(k)=C_{\text {aug }} x_{\text {aug }}(k) .
\end{array}
$$

From the above system of equations the original 8 by 8 pendulum system can be regenerated. The original system is just the 8 by 8 block part of $\left(A_{\text {aug }}-B_{\text {aug }} K_{\text {mpc }}\right)$ and the first 8 lines of $B_{\text {aug }}$. The block diagram of the MPC problem is shown in Fig. 3.

\section{SIMULATION RESULTS}

The parameters used in this work for the system illustrated in section $\mathbf{2}$ are addressed in table 1, the parameters are calculated from the CAD model designed in Solidworks software; the design process is as follow:

- $\quad$ Build 3D model for each element cart, first pendulum, etc,

- $\quad$ Add material property for each element,

- Calculate the mass, center of mass, and inertia for each element, and finally 
- Assemble the elements together to form the triple inverted-pendulum system shown in Fig. 1.

In table $1 ; m_{0}, m_{1}, m_{2}$, and $m_{3}$ are the masses of cart, first pendulum, second pendulum, and third pendulum respectively. The density of the material used in the design is $\rho=2700 \mathrm{~kg} / \mathrm{m}^{3}$. These masses are calculated automatically using the CAD system, the values of the dynamic moment of inertia $I_{1}, I_{2}$ and $I_{3}$ are also calculated in the CAD system then transferred to LabVIEW software. Using the parameters in the table 1, the model parameter $M_{i}$ 's values (9) are calculated and presented (table 2). All the dynamic model analysis and control design problem are solved using the graphical programming LabVIEW software.

The state feedback controller gains (11) calculated based on the linearized model (10) using the solution of Riccati equation (Error! Reference source not found.) are:

$K_{s}=[-316.23-2174.29157 .85-13115.81-377.71-845.51-646.30-1725.56]$, the observer gains $L_{s}$ in equation (12) are detailed in the appendix for the sake of shorten the presented numbers. The MPC gains produced using Laguerre function (19-21) are:

$K_{m p c}=[-60442.05-254453.23203167 .12-698366.46-34158.92-74959.33-$ $63720.31-93792.52]$

The designed controllers (State feedback and Model Predictive) first tested against the initial conditions $y(t)=\left[r \vartheta_{1} \gamma_{1} \gamma_{2}\right]^{T}=\left[\begin{array}{lll}-0.320^{\circ} 20^{\circ} 0 & 0\end{array}\right]$ for these initial conditions without controllers the pendulums will fall. In figure 4, the controlled motion of the system due to initial conditions is presented, it can be noticed from the figure that both state feedback and MP controllers are successfully generated the required control input to stabilize the system (all the outputs equal zero at the end of the simulation). The only one advantage for the MP controller over the state feedback controller, is the faster settling time. In order to test the controllers under complicated situation, three suggested paths for the cart are introduced as follow,

- A sinusoidal motion for 10 seconds then constant value:

$$
r_{d}(t)=\left\{\begin{array}{cc}
0.5 \sin (t) & t \leq 10 \\
0.5 & t>10
\end{array}\right.
$$

- A sinusoidal motion distorted with a random function:

$$
r_{d}(t)=0.4 \sin (t)+\operatorname{random}(t) .
$$

- A sinusoidal motion distorted with a random function for 14 seconds then minus constant value:

$$
r_{d}(t)=\left\{\begin{array}{cc}
0.4 \sin (t)+\operatorname{random}(t) & t \leq 14 \\
-0.35 & t>14
\end{array}\right.
$$

For the sake of the simulation the suggested paths are combined in the desired motion states as:

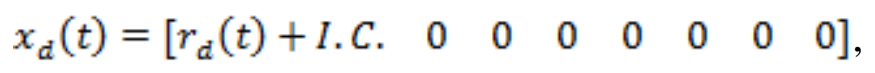

here $I . C$. is the initial condition of the cart position.

The simulation results for the suggested paths are presented in Figs. (5-10). First the state feedback control for (22) as desired cart motion is shown in Fig. 5, and the input control force generated from tee feedback law is shown in figure 6, it is clear that the state feedback control is 
capable of following the cart motion and keep the system stable in the vertical position "preventing the pendulums from falling". The MPC is used with both of (23 and 24) paths, the results are addressed in Figs. (7-10). Model predictive control is completely successful of determining the required force to avoid the falling of the pendulum, even in the presence of the sinusoidal motion and the random effects in the desired input for the cart. Furthermore, the system is simulated in 3D environment by combining the LabVIEW system with Solidworks to verify effectiveness and reliability of the used control approaches. A set of videos are available online at the links in table 3.

\section{CONCLUSIONS}

The results of theoretical model and CAD design of triple inverted-pendulums system were analyzed. Two control scenarios were implemented theoretical simulation and in virtual simulation. The nonlinear mathematical model of the system was developed by integration the model of the cart and triple pendulums. The system equations of motion are solved and simulated using LabVIEW software; besides, a virtual 3D simulation in Solidworks CAD software. Afterwards, two control methodologies were applied to control the motion of the system. The derived mathematical model was successfully validated using the two control techniques presented. The CAD model represents a good simplification for the dynamic model design and control. Virtual simulation addressed in this work signifies the validation process in modeling and control of complex mechanical system. Further work is necessary in the directions of CAD modeling and control.

Table 1. Systems parameters using Solidworks.

\begin{tabular}{|c|c|c|c|c|c|c|c|}
\hline Parameter & Unit & Parameter & Unit & Parameter & Unit & Parameter & Unit \\
\hline$m_{0}=1.521$ & $\mathrm{~kg}$ & $L_{1}=0.3$ & $m$ & $l_{1}=0.142$ & $m$ & $I_{1}=0.0127$ & $\mathrm{~kg} \times \mathrm{m}^{2}$ \\
\hline$m_{1}=0.433$ & $\mathrm{~kg}$ & $L_{2}=0.25$ & $m$ & $l_{2}=0.117$ & $m$ & $I_{2}=0.0077$ & $\mathrm{~kg} \times \mathrm{m}^{2}$ \\
\hline$m_{2}=0.375$ & $\mathrm{~kg}$ & $L_{3}=0.2$ & $m$ & $l_{3}=0.093$ & $m$ & $I_{3}=0.004$ & $k g \times m^{2}$ \\
\hline$m_{3}=0.307$ & $\mathrm{~kg}$ & & & & & & \\
\hline
\end{tabular}


Table 2. The model parameter $M_{i}$ 's values calculated based on the inertial properties from Solidworks.

\begin{tabular}{|l|l|l|l|}
\hline$M_{1}=2.6369$ & $M_{10}=0.0828$ & $M_{19}=0.0362$ & $M_{28}=0.0285$ \\
$M_{2}=0.2661$ & $M_{11}=0.0362$ & $M_{20}=0.0363$ & $M_{29}=0.0085$ \\
$M_{3}=0.1207$ & $M_{12}=0.0085$ & $M_{21}=0.0071$ & $M_{30}=0.0071$ \\
$M_{4}=0.0285$ & $M_{13}=0.000537$ & $M_{22}=-0.0362$ & $M_{31}=0.0066$ \\
$M_{5}=5.52$ & $M_{14}=0.0362$ & $M_{23}=-0.000269$ & $M_{32}=0.000469$ \\
$M_{6}=-0.2661$ & $M_{15}=-0.000269$ & $M_{24}=0.000537$ & $M_{33}=-0.0085$ \\
$M_{7}=-0.1207$ & $M_{16}=0.0085$ & $M_{25}=0.0071$ & $M_{34}=-0.2791$ \\
$M_{8}=-0.0285$ & $M_{17}=-2.6107$ & $M_{26}=-0.000269$ & $M_{35}=-0.0085$ \\
$M_{9}=0.2661$ & $M_{18}=0.1207$ & $M_{27}=-1.1839$ & $M_{36}=-0.000269$ \\
\hline
\end{tabular}

Table 1. 3D simulation Videos

\begin{tabular}{|l|l|}
\hline Free Fall & https://youtu.be/TxnSTxig0wE \\
\hline Triple Inverted-Pendulum State Feedback Control & https://youtu.be/TdSea9eV8H0 \\
\hline Triple Inverted-Pendulum MPC 2D view & https://youtu.be/ySO9TKJzYiQ \\
\hline Triple Inverted-Pendulum MPC 3D View & https://youtu.be/Rbr_5_M0u1o \\
\hline
\end{tabular}

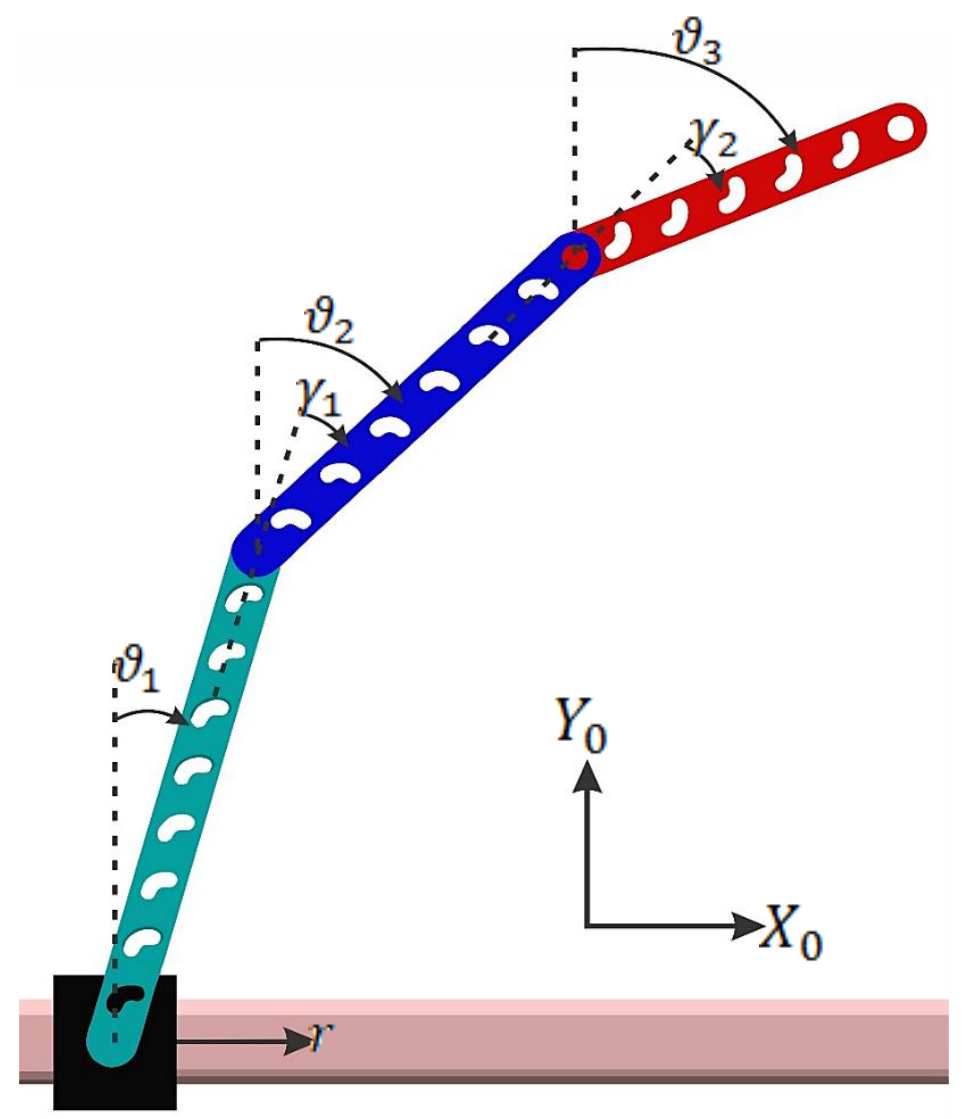

Figure 1. Triple Inverted-Pendulum Solidwroks Model. 


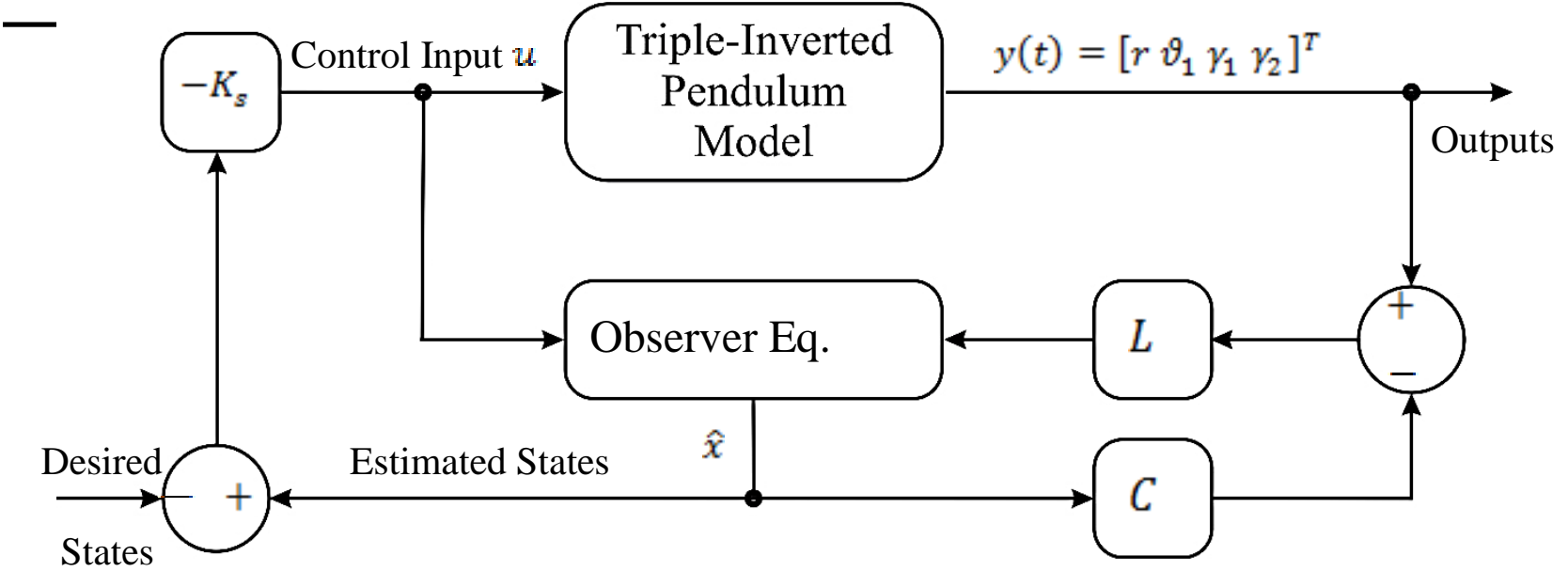

Figure 2. State feedback and observer problem diagram.

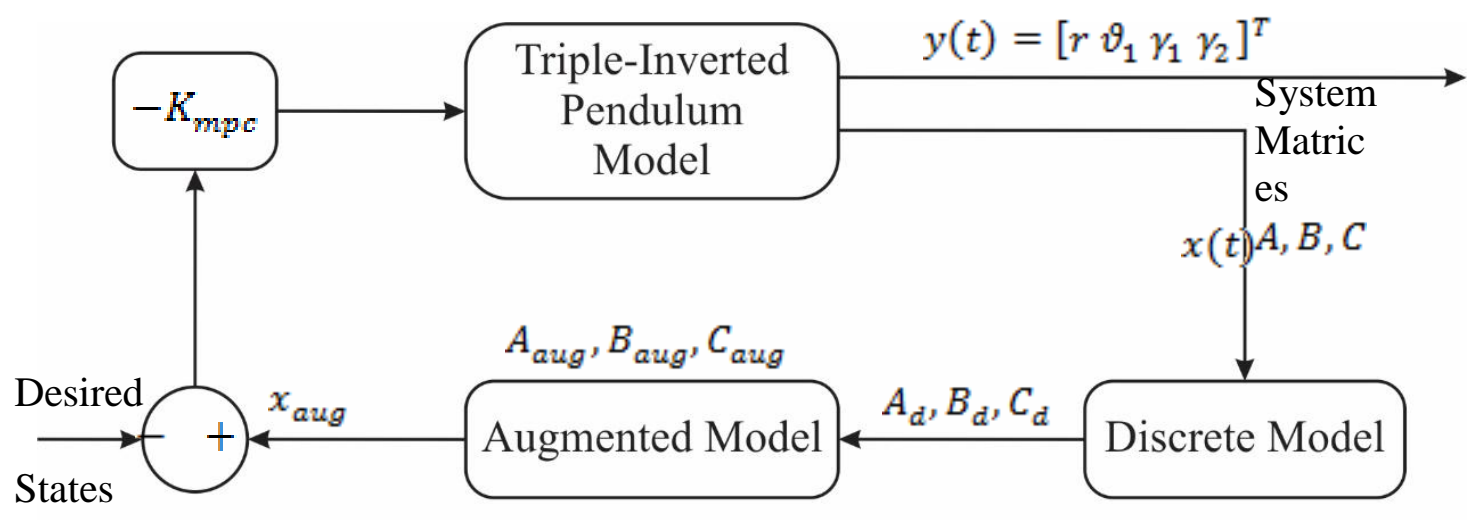

Figure 3. Model Predictive Control MPC problem diagram.
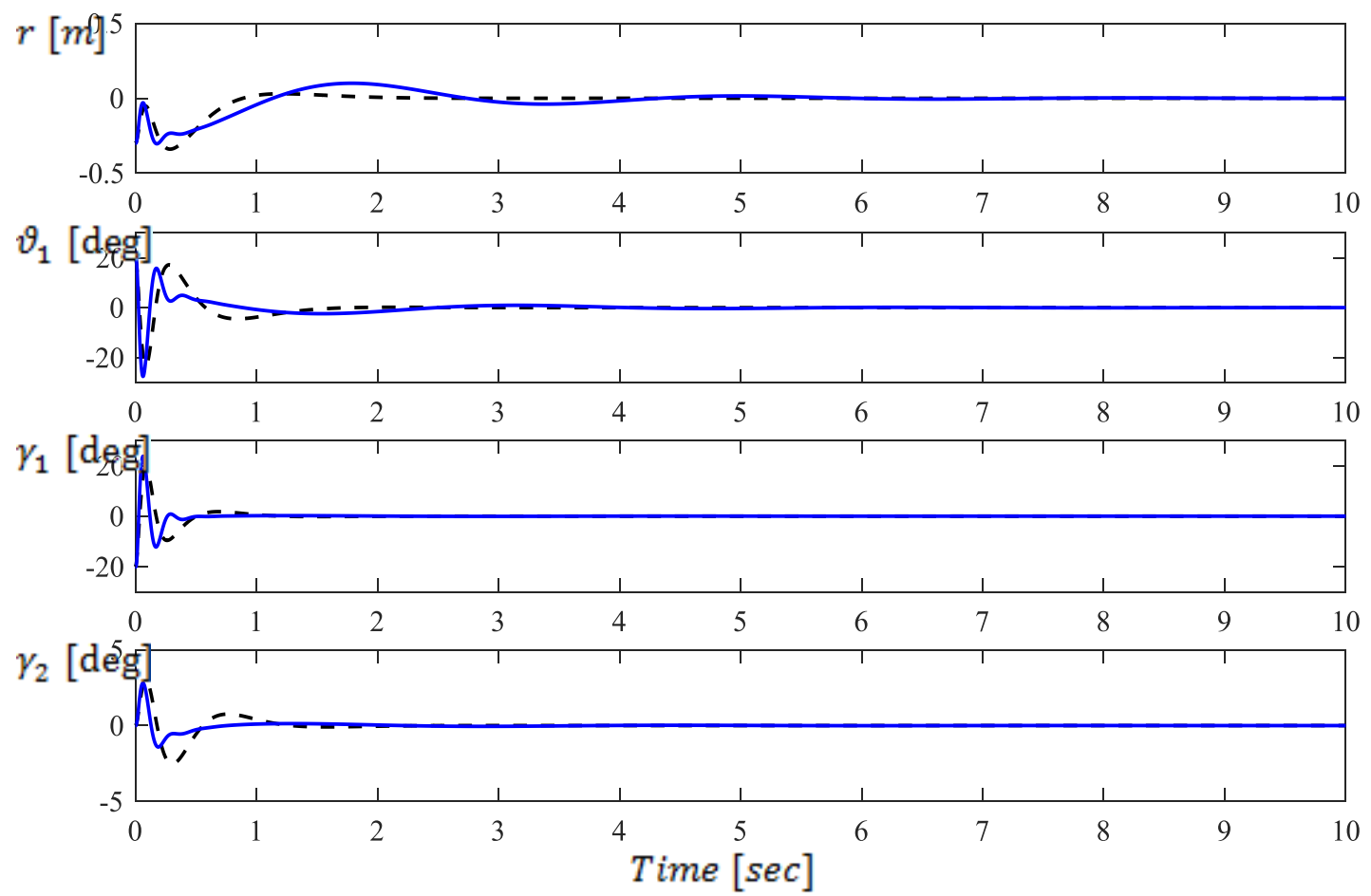

Figure 4. State feedback response - - - , and MPC response , due to initial conditions. 

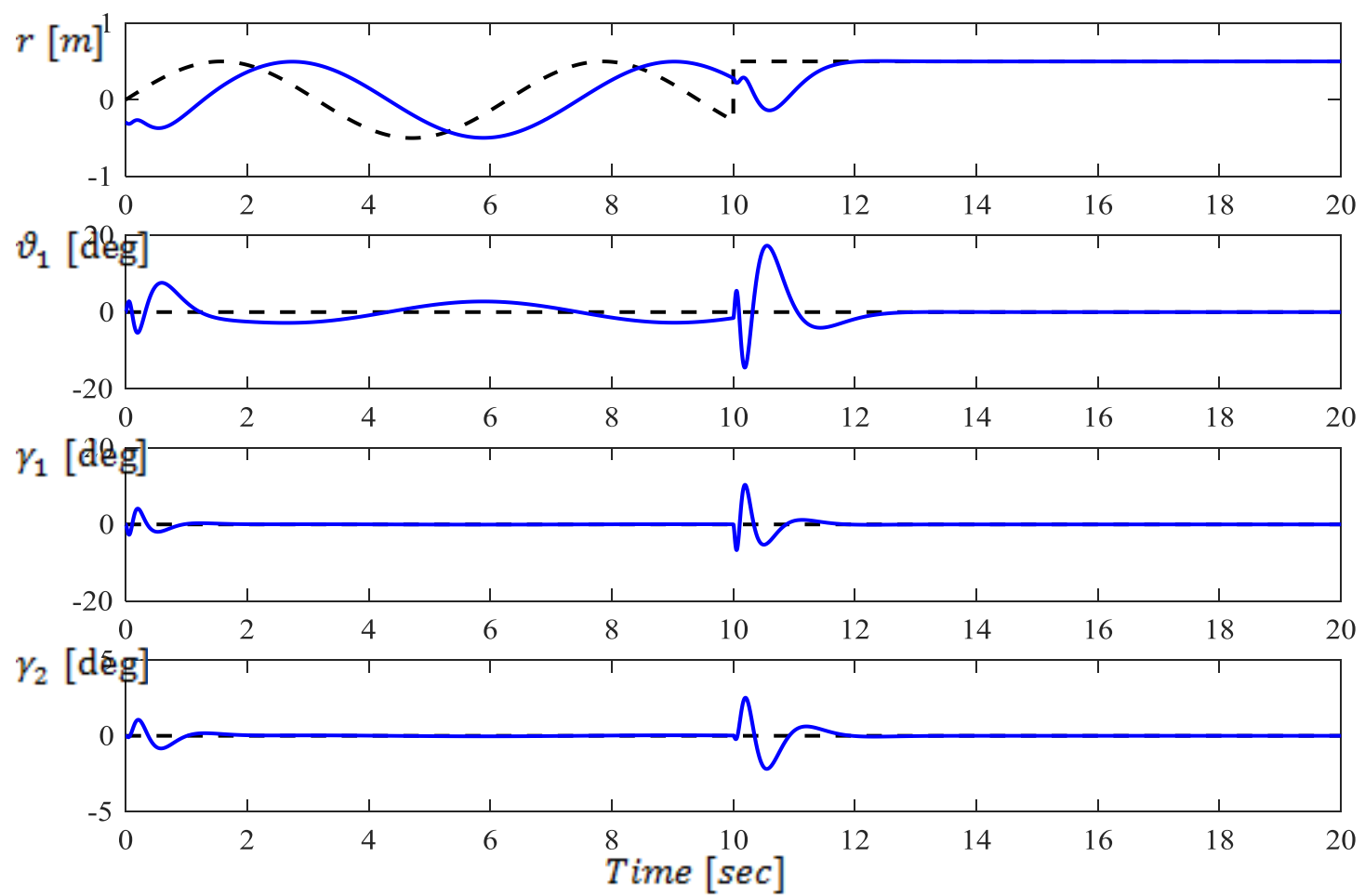

Figure 5. Desired response (Errer!-Reference source not found.) and State Feedback response , with initial conditions.

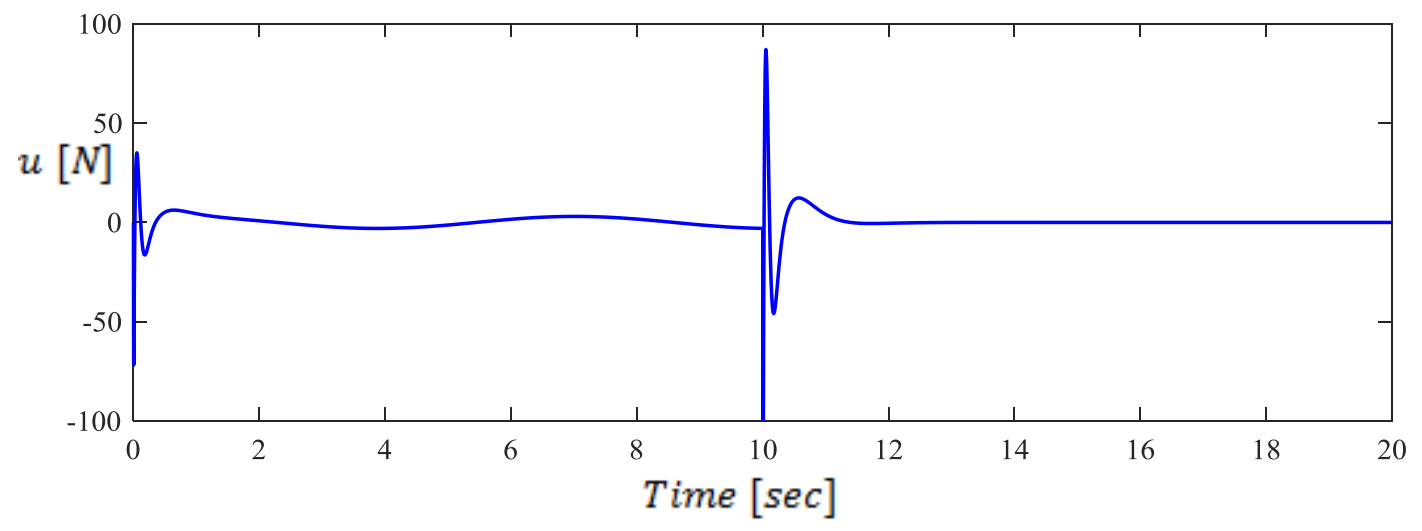

Figure 6. State Feedback Control input force. 


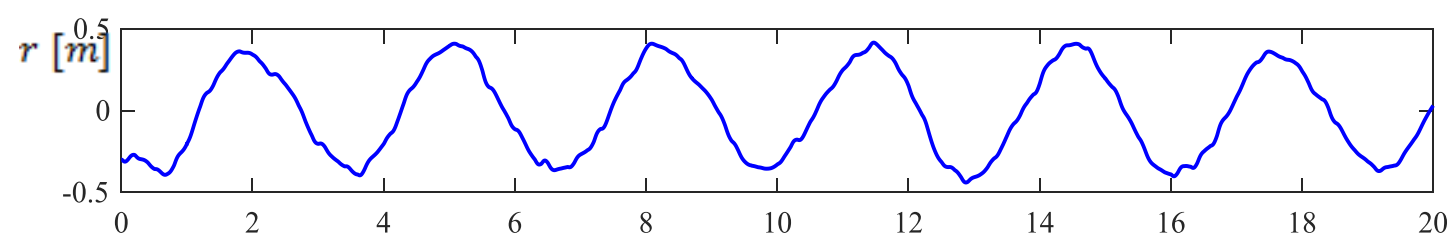

$v_{1}^{\vartheta_{1}}$

${ }_{-5}^{\gamma_{1}}$

$\gamma_{2}$ [deg]

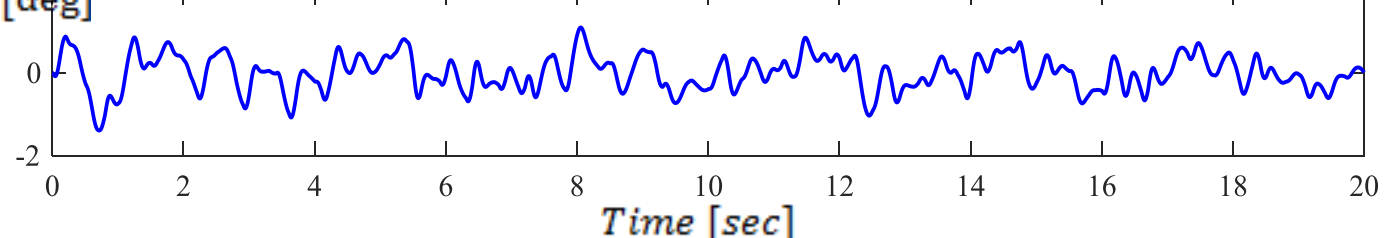

Figure 7. Model Predictive Control response due to random disturbance (23).

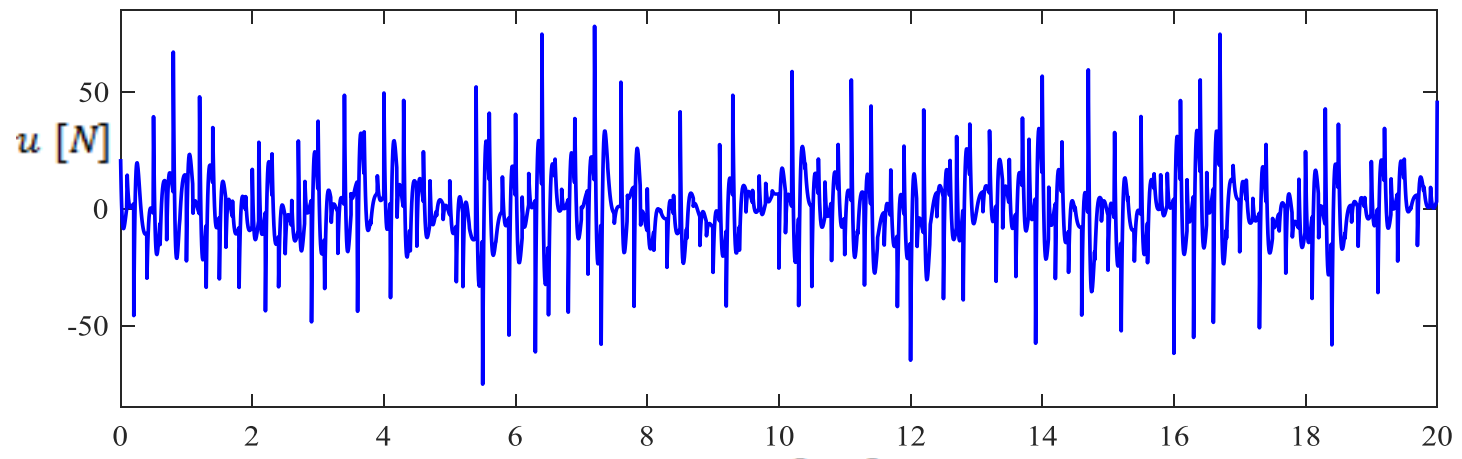

$r[m]$

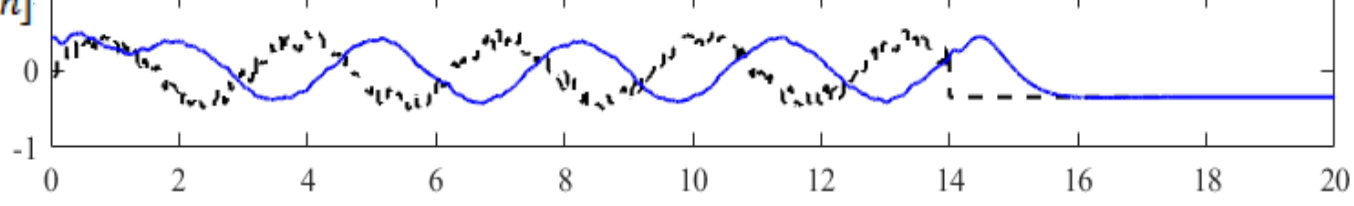

$$
\vartheta_{1}
$$

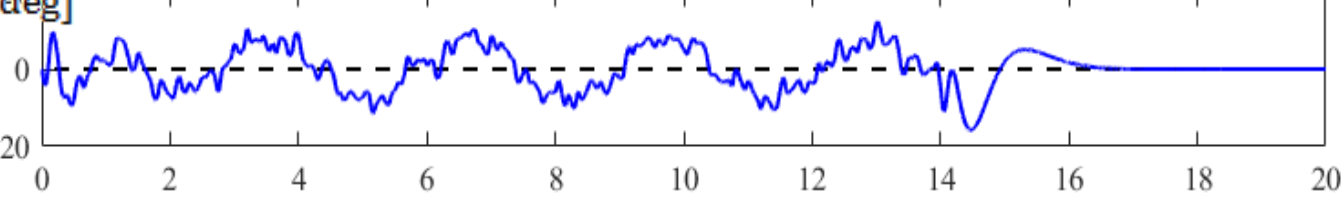

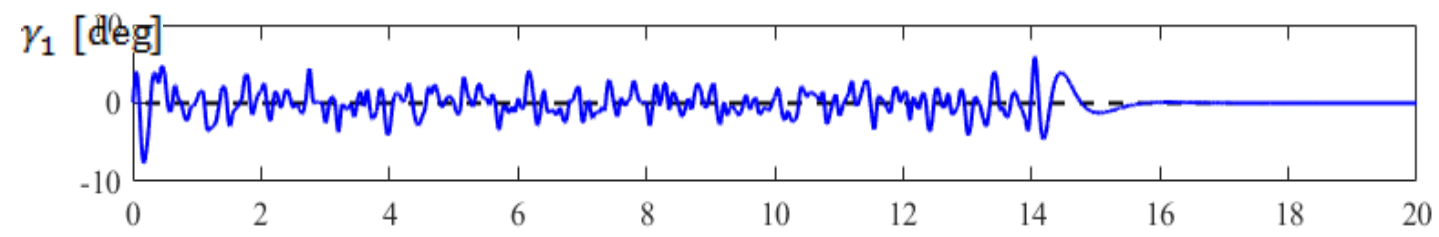

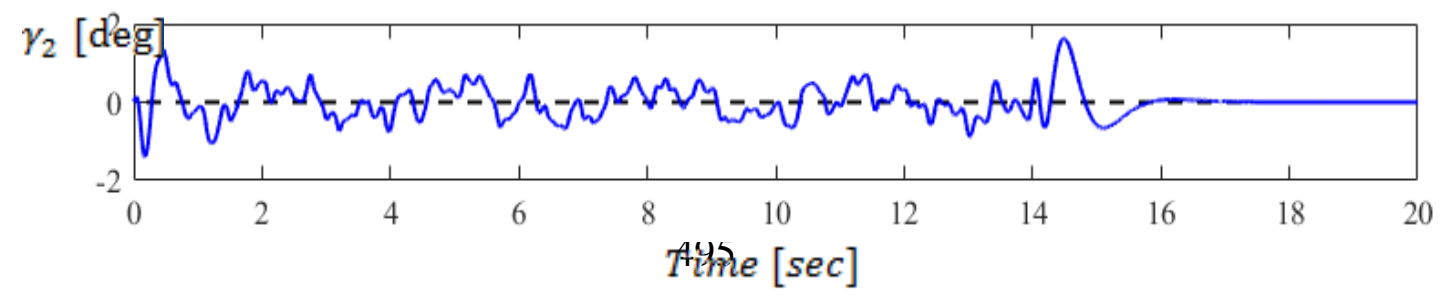

Figure 9. Model Predictive Control response for tracking a specific path (24). 


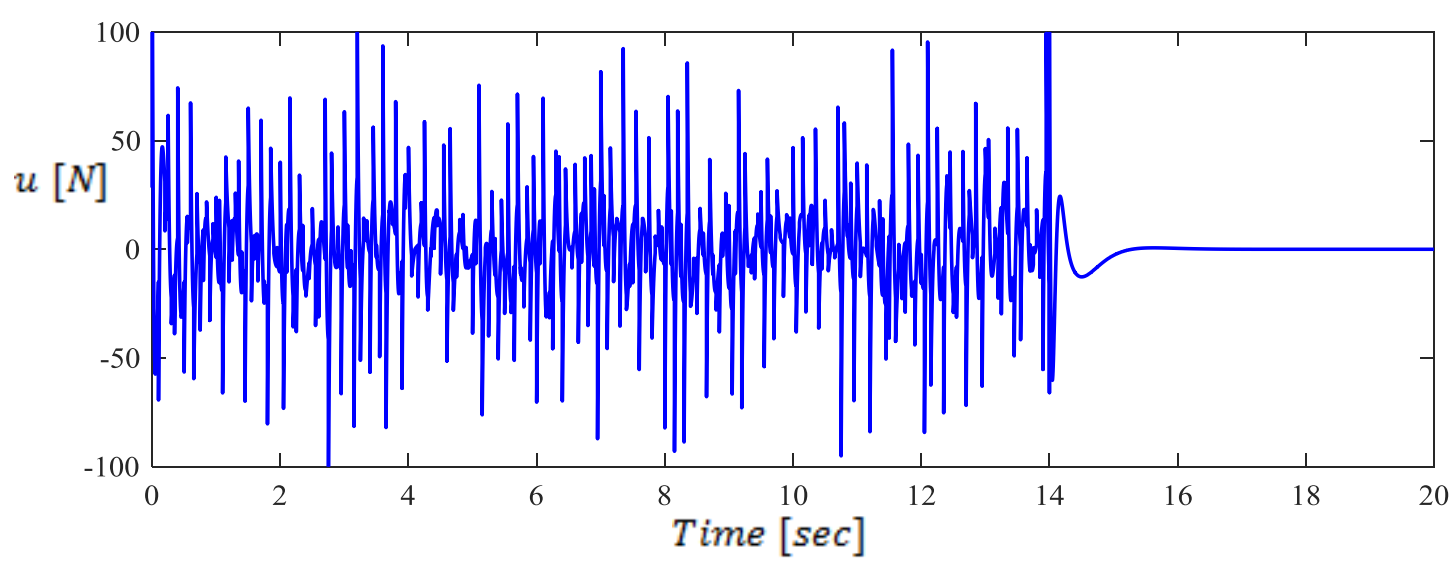

Figure 10. Model Predictive Control input force for tracking path (24).

\section{REFERENCES}

R. M. Brisilla and V. Sank, "Nonlinear Control of Mobile Inverted Pendulum", Robotics and Autonomous Systems, Vol. 70, pp. 145-155, 2015.

Jia-JunWang, "Simulation studies of inverted pendulum based on PID controllers" Simulation Modelling Practice and Theory, Vol. 19(1), pp. 440-449, 2011.

Ahmad Ilyas Roose, Samer Yahya, and Hussain Al-Rizzo, "Fuzzy-Logic Control of An Inverted Pendulum on a Cart", Computers \& Electrical Engineering Vol. 61, pp. 31-47, 2017.

H. Su and C.A. Woodham, "On The Uncontrollable Damped Triple Inverted-Pendulum”, Journal of Computational and Applied Mathematics 151, pp. 425-443, 2003.

Alexander Gmiterko and Martin Grossman, "An n-Link Inverted Pendulum Modeling", Acta Mechanica Slovaca Vol. 13(3), pp. 22-29, 2009.

Tobias Glück, Andreas Eder, and Andreas Kugi, "Swing-Up Control of a Triple Pendulum on a Cart with Experimental Validation”, Automatica Vol. 49, pp. 801-808, 2013.

Xiu-Ling Zhang, Hong-Min Fan, Jia-Yin Zang, Liang Zhao, and Shuang Hao, "The Stabilization and 3D Visual Simulation of the Triple Inverted Pendulum based on CGA-PIDNN", International Journal of Control, Automation, and Systems, Vol.13(4), pp. 1-10, 2015.

A. Coronel-Escamilla, J.F. Gómez-Aguilar, M.G. López-López , V.M. Alvarado-Martínez , and G.V. Guerrero-Ramírez, "Triple Pendulum Model Involving Fractional Derivatives With Different Kernels", Chaos, Solitons and Fractals Nonlinear Science, and Non-equilibrium and Complex Phenomena, Vol. 91, pp. 248-261, 2016.

V.A. Tsachouridis, "Robust Control of a Triple Inverted Pendulum", IEEE International Conference on Control Applications, 1999, Proceedings of the 1999, USA.

GW. Gu, Petkov P.H., and Konstantinov M.M., "A Triple Inverted Pendulum Control System Design. In: Robust Control Design with MATLAB®, Advanced Textbooks in Control and Signal Processing. Springer, London, 2013. 
Gene F. Franklin J. Da Powell and Abbas Emami-Naeini, "Feedback Control of Dynamic Systems" $7^{\text {th }}$ Global Edition, Pearson Higher Ed USA, 2014.

Richard C. Dorf and Robert H. Bishop, "Modern Control Systems", $13^{\text {th }}$ Glaobal Edition, Pearson Higher Ed USA, 2016.

Liuping Wang, "Model Predictive Control System Design and Implementation Using MATLAB", Springer, 2009.

Takashi Ohhira and Akira Shimada, "Model Predictive Control for an Inverted-pendulum Robot with Time-varying Constraints”, IFAC-PapersOnLine Vol. 50(1), pp. 776-781, 2017.

\section{APPENDIX}

Full State Observer Matrix

$L=\left[\begin{array}{cccc}632.455 & -0.00603 & -0.00029 & -0.000133 \\ -0.00603 & 137.861 & -13.213 & -11.887 \\ -0.000287 & -13.213 & 213.259 & -39.913 \\ -0.000133 & -11.887 & -39.913 & 487.212 \\ 0.9953 & 18.374 & -7.663 & -9.8705 \\ 0.00077 & 80.429 & 16.577 & 37.621 \\ -0.000384 & -45.2955 & 79.881 & 61.058 \\ 0.000922 & -89.535 & -298.642 & 320.939\end{array}\right]$

